\title{
Pancreatic Serous Cystadenocarcinoma
}

National Cancer Institute

\section{Source}

National Cancer Institute. Pancreatic Serous Cystadenocarcinoma. NCI Thesaurus. Code C5724.

A metastasizing, slow-growing malignant epithelial neoplasm that arises from the exocrine pancreas. It is characterized by the presence of cysts and is composed of glycogen-rich malignant epithelial cells which produce a watery fluid. Signs and symptoms include upper gastrointestinal bleeding, weight loss, jaundice, and abdominal pain. 